\title{
On Annihilating - Ideal Graph of $\mathbf{Z}_{\mathbf{n}}$
}

Husam Q. Mohammad

Sahbaa A. Younus

husam_alsabawi@yahoo.com

College of Computer Sciences and Mathematics

University of Mosul, Mosul, Iraq

\section{Received on: 14/05/2018}

Accepted on: 27/08/2018

\section{ABSTRACT}

In this paper, we study and give some properties of annihilating-ideal graphs of $\mathrm{Z}_{\mathrm{n}}$, also we find Hosoya polynomial and Wiener index for this graph.

Key word: Annihilating - ideal graph, $Z_{n}$, Hosoya polynomial, Wiener index.

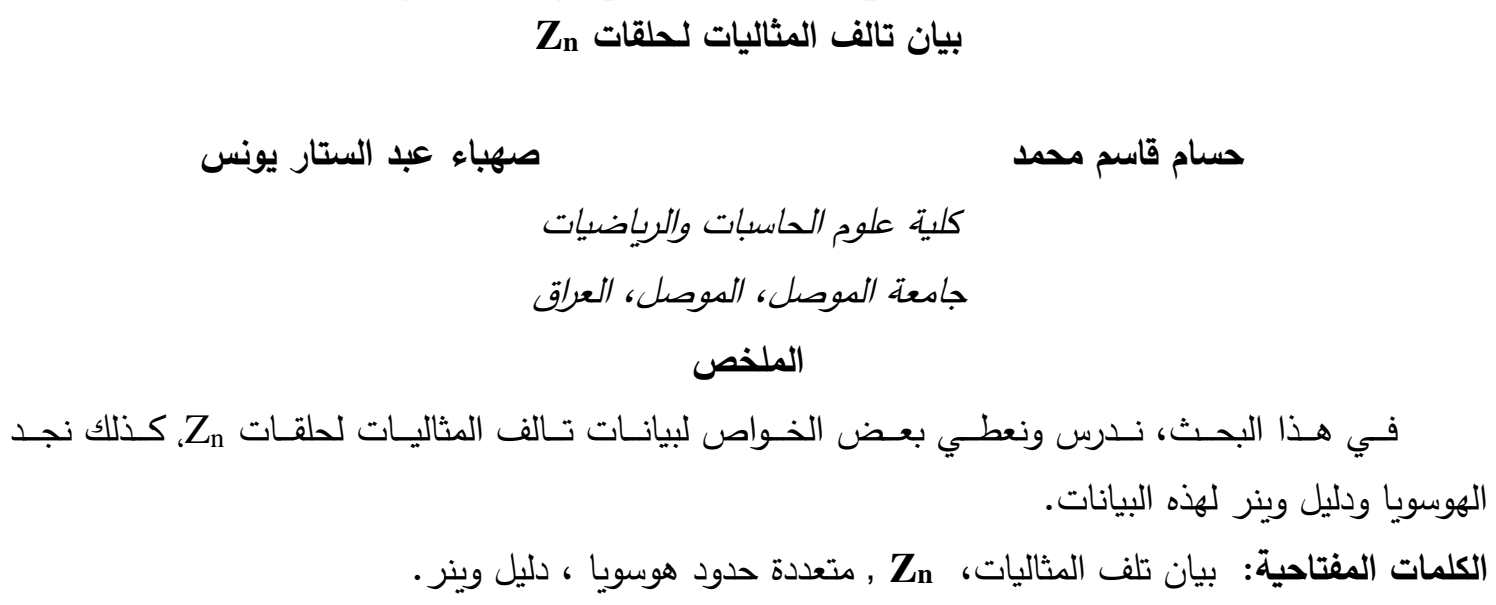

\section{Introduction}

In this paper $(\alpha)$ be the ideal of R generated by $\alpha$ and" $A^{*}(R)$ be the set of nonzero ideals with non-zero annihilators. We associate a simple graph $A G(R)$ with vertices $A^{*}(R)$ and two ideal vertices $I_{1}$ and $I_{2}$ are adjacent if and only if $I_{1} I_{2}=(0)[2] "$.

Recall according to [3]

1- Let $G(V, E)$ be a simple graph with vertices set $V$ and edges set $E$ be connected if there is a path between any two distinct vertices of G. For a vertices $x$ and $y$ of $G$, denoted $d(x, y)$ be the length of a shortest path form $x$ to $y$.

2- The diameter of $G$ denoted by $\operatorname{diam}(G)$ and equal $\max \{d(x, y)$ : $x$ and $y$ are vertices of $\mathrm{G}$ \}.

3- The degree of $x \in V(G)$ is denoted by $\operatorname{deg}(x)$ and it is the number of vertices who's adjacent with $\mathrm{x}$ in $\mathrm{G}$.

4- If $G_{1}$ and $G_{2}$ are graphs, then we say that $G_{1}$ is an isomorphic to $\mathbf{G}_{2},\left(\right.$ or $\left.G_{1} \cong G_{2}\right)$, if there exists a one-to -one mapping $\varphi$ from $V\left(G_{1}\right)$ onto $V\left(G_{2}\right)$ such that $\varphi$ preserves the adjacent.

5- The complete sub-graph $K_{t}$ of any graph $G$ is called a clique, and $\omega(\mathbf{G})$ is the clique number of $\mathbf{G}$, which is the greatest integer $\mathrm{t} \geq 1$, such that $\mathrm{K}_{\mathrm{t}} \subseteq \mathrm{G}$.

As usual, we shall assume that $\mathrm{p}$ and $\mathrm{q}$ are distinct prime numbers. $\lceil\mathrm{s}\rceil(\lfloor\mathrm{s}\rfloor$ resp.) It means that the smallest integer is not less than $\mathrm{s}$ (the greatest integer is not greater than $\mathrm{s}$ resp.). $Z_{n}$ we denote a ring of integer modulo $n$, . By [4] any ideal of $Z_{n}$ is principal and $Z_{n}$ local ring if and only if $n=p^{m}$, where $m$ is a positive integer number. In [2] Behboodi and Rakeei show that for every ring $R$, the annihilating-ideal graph $A G(R)$ is connected and $\operatorname{diam}(\mathrm{AG}(\mathrm{R})) \leq 3$. 


\section{2. on Annihilating-Ideal Graph of $\mathbf{Z}^{\mathrm{m}}$.}

In this section we give some basic properties of annihilating ideal graph of $Z_{\mathrm{P}}{ }^{\mathrm{m}}$. First we give an order and size of $A G\left(Z_{p}{ }^{m}\right)$, where $m \geq 4$.

\section{Theorem 2.1:}

If $\mathrm{R}=\mathrm{Z}_{\mathrm{p}}{ }^{\mathrm{m}}$, then $\mathrm{AG}(\mathrm{R})$ has order $\mathrm{m}-1$ vertices and the size $\mathrm{a}_{1}$, where

$\mathrm{a}_{1}= \begin{cases}\frac{\mathrm{m}(\mathrm{m}-2)}{4}, & \text { if m even, } \\ \frac{(\mathrm{m}-1)^{2}}{4}, & \text { if m odd. }\end{cases}$

Proof: Since any ideal of $Z_{n}$ is principal, then clearly that the ideals of ring $Z_{p}{ }^{m}$ $\operatorname{are}\left\{(\mathrm{p}),\left(\mathrm{p}^{2}\right), \ldots,\left(\mathrm{p}^{\mathrm{m}-1}\right)\right\}$, therefor $\mathrm{AG}(\mathrm{R})$ has $(\mathrm{m}-1)$ vertices ideal.

Now to find size of graph $A G(R)$.

Since $p^{m} \equiv 0 \bmod p^{m}$, then $\left(p^{i}\right)\left(p^{j}\right)=0$ iff $i+j \geq m$, where $1 \leq i, j \leq m-1$, so that $\left(p^{i}\right)$ adjacent with $\left(\mathrm{p}^{\mathrm{j}}\right)$ whenever $1 \leq \mathrm{i}<\mathrm{m}-1$ and $\mathrm{j}=(\mathrm{m}-\mathrm{i}), \ldots, \mathrm{m}-1$, which implies that

$\sum_{j=m-i}^{m-1} 1=(m-1)-(m-i)+1=i$, and since $\left(p^{i}\right)\left(p^{i}\right)=0$ if and only if $i \geq\left\lceil\frac{m}{2}\right\rceil$.

Then we have $\left(\mathrm{p}^{\mathrm{i}}\right)$ has loop, so that

$\operatorname{deg}\left(\left(p^{i}\right)\right)=\left\{\begin{array}{ccc}\mathrm{i}, & \text { if } & 1 \leq \mathrm{i} \leq\left\lceil\frac{\mathrm{m}}{2}\right\rceil-1 \\ \mathrm{i}-1, & \text { if } & \left\lceil\frac{\mathrm{m}}{2}\right\rceil \leq \mathrm{i} \leq \mathrm{m}-1\end{array}\right.$

and

$\sum_{v \in A G\left(z_{p} m\right)} \operatorname{deg} v=\sum_{i=1}^{\left\lceil\frac{\mathrm{m}}{2}\right\rceil-1} \mathrm{i}+\sum_{\mathrm{i}=\left\lceil\frac{\mathrm{m}}{2}\right\rceil}^{\mathrm{m}-1}(\mathrm{i}-1)=\sum_{\mathrm{i}=1}^{\mathrm{m}-1} \mathrm{i}-\sum_{\mathrm{i}=\left\lceil\frac{\mathrm{m}}{2}\right\rceil}^{\mathrm{m}-1} 1=\frac{\mathrm{m}(\mathrm{m}-3)}{2}+\left\lceil\frac{\mathrm{m}}{2}\right\rceil$

Since $\sum_{v \in A G\left(z_{p} m\right)} \operatorname{deg} v=2 a_{1}$, where $a_{1}$ is the number of edges of $A G\left(Z_{p}{ }^{m}\right)$, then $\mathrm{a}_{1}=\frac{\mathrm{m}(\mathrm{m}-2)}{4}$, when $\mathrm{m}$ is even, and $\mathrm{a}_{1}=\frac{(\mathrm{m}-1)^{2}}{4}$, when $\mathrm{m}$ is odd.

The next result we give the clique number of $\mathrm{AG}\left(\mathrm{Z}_{\mathrm{p}}{ }^{\mathrm{m}}\right)$.

\section{Theorem 2.2}

For any positive number $\mathrm{m} \geq 4, \mathrm{AG}\left(\mathrm{Z}_{\mathrm{p}}{ }^{\mathrm{m}}\right)$ contains a sub-graph $\mathrm{K}_{\left\lceil\frac{\mathrm{m}}{2}\right\rceil}$ and $\omega\left(\mathrm{AG}\left(\mathrm{Z}_{\mathrm{p}}{ }^{\mathrm{m}}\right)\right)=\left\lceil\frac{\mathrm{m}}{2}\right\rceil$

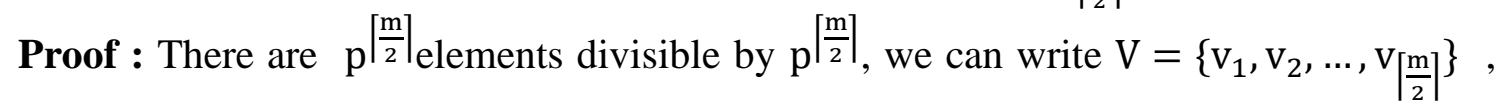
where $v_{i}=\left(p^{m-i}\right), 1 \leq \mathrm{i} \leq\left\lceil\frac{\mathrm{m}}{2}\right\rceil$ this means that all ideal vertices of $\mathrm{V}$ are adjacent each other . Thus $\mathrm{V}=\mathrm{V}\left(\mathrm{k}_{\left[\frac{\mathrm{m}}{2}\right]}\right)$ a complete sub graph of $\mathrm{AG}\left(\mathrm{Z}_{\mathrm{p}}{ }^{\mathrm{m}}\right)$. Also any ideal vertices $\mathrm{x}=\left(\mathrm{p}^{\mathrm{m}-\mathrm{i}}\right),\left\lceil\frac{\mathrm{m}}{2}\right\rceil+1 \leq \mathrm{i} \leq \mathrm{m}-1$ are non-adjacent with ideal vertex $\mathrm{v}_{\left\lceil\frac{\mathrm{m}}{2}\right\rceil}=\left(\mathrm{p}^{\left\lceil\frac{\mathrm{m}}{2}\right\rceil}\right)$, so that,$\left\lceil\frac{\mathrm{m}}{2}\right\rceil$ greats integer such that $\mathrm{k}_{\left\lceil\frac{\mathrm{m}}{2}\right\rceil} \subseteq \mathrm{AG}\left(\mathrm{Z}_{\mathrm{p}}{ }^{\mathrm{m}}\right)$, whence $\omega\left(\mathrm{AG}\left(\mathrm{Z}_{\mathrm{p}}{ }^{\mathrm{m}}\right)\right)=\left\lceil\frac{\mathrm{m}}{2}\right\rceil$

Recall that "radius of $G$ is $\operatorname{rad}(G)=\min \{d(x, y): x$ and $y$ are vertices of $G\}$ and the center of $G$ is defined by $\operatorname{Cent}(G)=\{x \in V(G): d(x, y)=\operatorname{rad}(G)$ for any $\mathrm{y} \in \mathrm{V}(\mathrm{G})\}[3] "$.

\section{Proposition2.3}

For any positive number $\mathrm{m} \geq 4, \operatorname{rad}\left(A G\left(Z_{p}{ }^{m}\right)\right)=1$ and $\operatorname{Cent}\left(A G\left(Z_{p}{ }^{m}\right)\right)=\left(p^{m-1}\right)$. 
Proof: Since $Z_{p}{ }^{m}$ be a local ring, then by [7] every minimal ideal vertex adjacent with every ideal vertices in $A G\left(Z_{p}{ }^{m}\right)$. That means that the graph $A G\left(Z_{p}{ }^{m}\right)$ contains subgraph

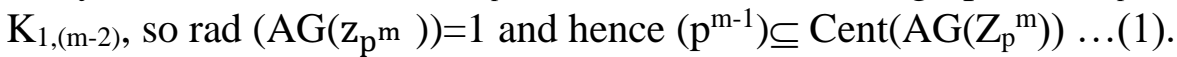

Now, let $x \in A G\left(Z_{p}{ }^{m}\right)$ such that $x \notin\left(p^{m-1}\right)$ and for any $y \in(p)$, then $x y \neq 0$, this means that $x \notin \operatorname{Cent}\left(A G\left(Z_{p}{ }^{m}\right)\right)$ and hence $\operatorname{Cent}\left(A G\left(Z_{p}{ }^{m}\right)\right) \subseteq\left(p^{m-1}\right) \ldots(2)$.

Form (1) and (2), we get Cent $\left(A G\left(Z_{p}{ }^{m}\right)\right)=\left(p^{m-1}\right)$

\section{Proposition 2.4}

For any positive number $m \geq 4$, we have $\operatorname{diam}\left(\mathrm{AG}\left(\mathrm{Z}_{\mathrm{p}}{ }^{\mathrm{m}}\right)\right)=2$

Proof: Clearly the ideal vertex $\left(\mathrm{p}^{\mathrm{m}-1}\right)$ adjacent with every ideal vertices in $\mathrm{AG}\left(\mathrm{Z}_{\mathrm{p}}{ }^{\mathrm{m}}\right)$, also the ideal vertices $\alpha_{1}=(p)$ and $\alpha_{2}=\left(p^{2}\right)$ are non-adjacent, then we have $\operatorname{diam}\left(A G\left(Z_{p}{ }^{m}\right)\right)=2$.

\section{Theorem 2.5}

For any positive number $\mathrm{m} \geq 4, \operatorname{AG}\left(\mathrm{Z}_{\mathrm{p}}{ }^{\mathrm{m}}\right) \cong\left(\operatorname{AG}\left(\mathrm{Z}_{\mathrm{p}}{ }^{\mathrm{m}-1}\right) \cup\left\{\mathrm{K}_{1}, \mathrm{U}_{\mathrm{s}}\right\}\right)$, where $\mathrm{s}=\left\lceil\frac{\mathrm{m}}{2}\right\rceil-1$ and $U_{s}$ is added edges are numbered $s$ that connecting $K_{1}$ to $\left(p^{m-i}\right)$ where $i=1,2, \ldots,\left\lfloor\frac{m}{2}\right\rfloor$.

Proof: We observe that the number of vertices of two graph are equal $(\mathrm{m}-1)$. In $\operatorname{AG}\left(Z_{p}{ }^{m}\right)$ the ideal vertices $\left(p^{i}\right)$ and $\left(p^{j}\right)$ are adjacent if and only if $i+j \geq m$, also in $\mathrm{G}=\left(\mathrm{AG}\left(\mathrm{Z}_{\mathrm{p}}{ }^{\mathrm{m}-1}\right) \cup\left\{\mathrm{K}_{1}, \mathrm{U}_{\mathrm{s}}\right\}\right)$, the ideal vertices $\mathrm{u}_{\mathrm{i}}=\left(\mathrm{p}^{\mathrm{i}}\right)$ and $\mathrm{u}_{\mathrm{j}}=\left(\mathrm{p}^{\mathrm{j}}\right)$ are adjacent if and only if $\mathrm{i}+\mathrm{j} \geq \mathrm{m}-1$, or $\mathrm{u}_{\mathrm{i}}=\mathrm{w}$, where $\mathrm{V}\left(\mathrm{K}_{1}\right)=\mathrm{w}, \mathrm{i}=\left\lceil\frac{\mathrm{m}}{2}\right\rceil$ and $\mathrm{j}=\left\lceil\frac{\mathrm{m}}{2}\right\rceil, \ldots, \mathrm{m}-2$. Therefor we can label the vertices in $\mathrm{AG}\left(\mathrm{Z}_{\mathrm{p}}^{\mathrm{m}}\right)$ are $\mathrm{v}_{\mathrm{i}}=\left(\mathrm{p}^{\mathrm{i}}\right), \mathrm{i}=1, \ldots, \mathrm{m}-1$ and the vertices in $\mathrm{G}$ are

$$
u_{i}=\left\{\begin{array}{cl}
\left(p^{i}\right), & \text { if } i=1,2, \ldots,\left\lceil\frac{m}{2}\right\rceil-1, \\
w, & \text { if } i=\left\lceil\frac{m}{2}\right\rceil, \\
\left(p^{i-1}\right), & \text { if } i=\left\lceil\frac{m}{2}\right\rceil+1, \ldots, m-1 .
\end{array}\right.
$$

We can defined a mapping $\mathrm{f}$ from $\mathrm{AG}\left(\mathrm{Z}_{\mathrm{p}}{ }^{\mathrm{m}}\right)$ to, $\mathrm{G}=\left(\mathrm{AG}\left(\mathrm{Z}_{\mathrm{p}}{ }^{\mathrm{m}-1}\right) \cup\left\{\mathrm{K}_{1}, \mathrm{U}_{\mathrm{s}}\right\}\right)$, such that $\mathrm{f}\left(\mathrm{v}_{\mathrm{i}}\right)=\mathrm{u}_{\mathrm{i}}$, where $1 \leq \mathrm{i} \leq \mathrm{m}-1$.

Clearly that $\mathrm{f}$ is onto and one-to-one, we only prove that if $\mathrm{e}=\mathrm{uv}$ is an edge in $\operatorname{AG}\left(Z_{p}{ }^{m}\right)$ then $f(e)=f(u) f(v)$ is an edge in $G$.

Let $e=v_{i} v_{j}$ edge from $v_{i}$ to $v_{j}$ in $A G\left(Z_{p}^{m}\right)$, therefore $i+j \geq m$ we get two cases.

Case 1: If $\mathrm{i}$ or $\mathrm{j}=\left\lceil\frac{\mathrm{m}}{2}\right\rceil$, say $\mathrm{i}=\left\lceil\frac{\mathrm{m}}{2}\right\rceil$ then $\mathrm{v}_{\mathrm{j}}$ has two sub cases $\mathrm{a}$ and $\mathrm{b}$ :

Sub-case a: If $m$ is an even number then $\left\lceil\frac{m}{2}\right\rceil=\frac{m}{2}$, so $v_{i}$ adjacent with $v_{j}$, where $\mathrm{j}=\left(\frac{\mathrm{m}}{2}+1\right), \ldots, \mathrm{m}-1$, on the other hand $\mathrm{i}=\frac{\mathrm{m}}{2}, \mathrm{f}\left(\mathrm{v}_{\mathrm{i}}\right)=\mathrm{w}$ and $\mathrm{f}\left(\mathrm{v}_{\mathrm{j}}\right)=\mathrm{u}_{\mathrm{j}}=\left(\mathrm{p}^{\mathrm{j}-1}\right)$ for all $\mathrm{j}=\left(\frac{\mathrm{m}}{2}+1\right)$ $, \ldots, m-1$. From defined $G$, we see $w$ adjacent with $u_{j}=\left(p^{j-1}\right), j=\frac{m}{2}+1, \ldots, m-1$, therefor if $e$ is an edge in $\operatorname{AG}\left(Z_{p}{ }^{m}\right)$, then $f(e)$ is an edge in $G$.

Sub-case b: If $m$ is odd then $\left\lceil\frac{m}{2}\right\rceil=\frac{m+1}{2}$, the vertex $v_{i}$ adjacent with $v_{j}$ if and only if $\mathrm{j}=\frac{\mathrm{m}-1}{2}, \frac{\mathrm{m}+1}{2}, \frac{\mathrm{m}+1}{2}+1, \ldots, \mathrm{m}-1$, except case, $\frac{(\mathrm{m}+1)}{2}$ because $\mathrm{i}=\mathrm{j}$, $\mathrm{v}_{\mathrm{i}}$ adjacent with $\mathrm{v}_{\mathrm{j}}$. Where $\mathrm{j}=\frac{(\mathrm{m}-1)}{2},\left(\frac{\mathrm{m}+1}{2}+1\right), \ldots, \mathrm{m}-1$. On the other hand since $\mathrm{i}=\left(\frac{\mathrm{m}+1}{2}\right)$ then $\mathrm{f}\left(\mathrm{v}_{\mathrm{i}}\right)=\mathrm{w}$ and 


$$
f\left(v_{j}\right)=\left\{\begin{array}{lll}
\left(p^{j}\right), & \text { if } & j=\frac{m-1}{2}, \\
\left(p^{j-1}\right), & \text { if } & j=\frac{m+1}{2}+1, \ldots, m-1 .
\end{array}\right.
$$

From defined $G$, observe that $w$ adjacent with $u_{j}, j=\frac{(m-1)}{2}, \frac{(m+1)}{2}+1, \ldots, m-2$. That mean if e is an edge in $\operatorname{AG}\left(Z_{p}{ }^{m}\right)$ then $f(e)$ is an edge in $G$.

Case 2: If $i$ and $j \neq\left\lceil\frac{m}{2}\right\rceil$, then e edge in $A G\left(Z_{p}{ }^{m}\right)$ iff $i+j \geq m$ and $i, j \neq\left\lceil\frac{m}{2}\right\rceil$.

We get two sub-cases $\mathrm{c}$ and $\mathrm{d}$ :

Sub-case c: if $\mathrm{i}$ or $\mathrm{j}<\left\lceil\frac{\mathrm{m}}{2}\right\rceil$, without loss generality let $\mathrm{i}<\left\lceil\frac{\mathrm{m}}{2}\right\rceil$, then $\mathrm{f}\left(\mathrm{v}_{\mathrm{i}}\right)=\mathrm{u}_{\mathrm{i}}=\left(\mathrm{p}^{\mathrm{i}}\right)$

since $i+j \geq m$ we get $j>\left\lceil\frac{m}{2}\right\rceil$, therefor $f\left(v_{j}\right)=\left(p^{j-1}\right)=u_{k}$, where $j=\left\lceil\frac{m}{2}\right\rceil+1, \ldots, m-1$ and $k=j-1$ on the other hand $u_{i} u_{k}$ adjacent iff $i+k \geq m-1$, because $p^{m-1} \equiv 0 \bmod m-1$, therefor $u_{i}$ adjacent with $u_{k}$ that mean if e is an edge in $A G\left(z_{p^{m}}\right)$, then $f(e)$ is an edge in $G$.

Sub-case $d$ : if $i$ and $j>\left\lceil\frac{m}{2}\right\rceil$, then $f\left(v_{i}\right)=u_{i}=\left(p^{i-1}\right)$ and $f\left(v_{j}\right)=u_{j}=\left(p^{j-1}\right)$,

since $i>\left\lceil\frac{m}{2}\right\rceil$ then $u_{i}, u_{j}$ adjacent where $i \neq j$ and $i, j=\left\lceil\frac{m}{2}\right\rceil, \ldots, m-1$ that is means, if $e$ is an edge in $A G\left(Z_{p}{ }^{m}\right)$, then $f(e)$ is an edge .

So that $A G\left(Z_{p}{ }^{m}\right) \cong G$

\section{On Annihilating- Ideal Graph of $Z_{p}{ }^{m} q$.}

First we give order and size of $A G\left(\mathbf{Z}_{\mathbf{p}}{ }^{\mathbf{m}} \mathbf{q}\right)$ for all positive number $\mathrm{m} \geq 3$.

\section{Theorem 3.1}

Let $R=Z_{p}{ }^{m}$, then $A G(R)$ has order $2 m$ vertices and the sizes

$$
a_{1}=\left\{\begin{array}{llr}
\frac{3 m^{2}}{4}, & \text { if } m \text { even }, \\
\frac{3 \mathrm{~m}^{2}+1}{4}, & \text { if } \quad \text { odd. }
\end{array}\right.
$$

Proof : The ideals of ring $R$ are $\left\{(p),\left(p^{2}\right), \ldots,\left(p^{m}\right),(q),(p q),\left(p^{2} q\right), \ldots,\left(p^{m-1} q\right)\right\}$. So that $\operatorname{AG}\left(\mathrm{Z}_{\mathrm{p}}{ }_{\mathrm{q}}\right)$ has $2 \mathrm{~m}$ vertices ideal.

Now to find size of graph $A G\left(Z_{p}{ }^{m}{ }_{q}\right)$, we must find degree of any vertices ideal.

Since $\mathrm{p}^{\mathrm{m}} \mathrm{q} \equiv 0 \bmod \mathrm{p}^{\mathrm{m}} \mathrm{q}$, and $\mathrm{p}$ and $\mathrm{q}$ are distinct prime, then $\left(\mathrm{p}^{\mathrm{i}}\right)$ is adjacent with $\left(\mathrm{p}^{\mathrm{j}} \mathrm{q}\right)$ iff $\mathrm{i}+\mathrm{j} \geq \mathrm{m}$, where $0 \leq \mathrm{j} \leq \mathrm{m}-1,1 \leq \mathrm{i} \leq \mathrm{m}$. Then

$\operatorname{deg}\left(\mathrm{p}^{\mathrm{i}}\right)=\sum_{\mathrm{j}=\mathrm{m}-\mathrm{i}}^{\mathrm{m}-1} 1=\mathrm{m}-1-(\mathrm{m}-\mathrm{i})+1=\mathrm{i}$. Also (q) adjacent with only ideal vertex $\left(\mathrm{p}^{\mathrm{m}}\right)$ so that $\operatorname{deg}(q)=1$. Finally since $\left(p^{i} q\right)$ is adjacent with $\left(p^{j}\right)$ iff $i+j \geq m$ and $\sum_{j=m-i}^{m} 1=i+1$. Also $\left(p^{i} q\right)$ is adjacent with $\left(p^{j} q\right)$ iff $i+j \geq m$, for all $1 \leq i, j \leq m-1$ and since $\sum_{j=m-i}^{m-1} 1=i$, then $\sum_{j=m-i}^{m-1} 1+\sum_{j=m-i}^{m} 1=2 i+1$, also, we note $\left(p^{i} q\right)$ contained loop if and only if $i \geq\left\lceil\frac{m}{2}\right\rceil$ Therefor

$$
\operatorname{deg}\left(p^{i} q\right)=\left\{\begin{array}{cc}
2 i+1, & \text { if } 1 \leq i<\left\lceil\frac{\mathrm{m}}{2}\right\rceil, \\
2 \mathrm{i}, & \text { if }\left\lceil\frac{\mathrm{m}}{2}\right\rceil \leq \mathrm{i} \leq \mathrm{m}-1,
\end{array}\right.
$$


Which implies that

$$
\begin{gathered}
\sum_{v \in A G(R)} \operatorname{deg} v=1+\sum_{i=1}^{\left\lceil\frac{m}{2}\right\rceil-1}(2 i+1)+\sum_{i=\left\lceil\frac{m}{2}\right\rceil}^{i=m-1} 2 i+\sum_{i=1}^{i=m} i \\
=1+\left\lceil\frac{m}{2}\right\rceil-1+\sum_{i=1}^{i=m-1} 2 i+\sum_{i=1}^{i=m} i=\left\lceil\frac{m}{2}\right\rceil+\frac{2(m-1) m}{2}+\frac{m(m+1)}{2} \\
=\left\lceil\frac{m}{2}\right\rceil+\frac{3 m^{2}-m}{2} .
\end{gathered}
$$

Since

$$
\sum_{v \in A G(R)} \operatorname{deg} v=2 a_{1}
$$

, where $\mathrm{a}_{1}$ is the number of edges of $\mathrm{AG}\left(\mathrm{Z}_{\mathrm{p}} \mathrm{m}_{\mathrm{q}}\right)$, then

and

$$
\mathrm{a}_{1}=\frac{3 \mathrm{~m}^{2}}{4}, \text { if } \mathrm{m} \text { even }
$$

$\mathrm{a}_{1}=\frac{3 \mathrm{~m}^{2}+1}{4}$, if $\mathrm{m}$ odd .

\section{Theorem 3.2}

for all positive number $\mathrm{m} \geq 3$. $A G\left(Z_{p}{ }^{m}\right)$ contains a sub-graph $K_{\left\lceil\frac{m}{2}\right\rceil+1}$ and $\omega(\mathrm{AG})=\left\lceil\frac{\mathrm{m}}{2}\right\rceil+1$

Proof: We can write $\left.\mathrm{V}=\left\{\mathrm{v}_{1}, \mathrm{v}_{2}, \ldots, \mathrm{v}_{\lceil} \frac{\mathrm{m}}{2}\right\rceil\right\}$, where $\mathrm{v}_{\mathrm{i}}=\left(\mathrm{p}^{\mathrm{m}-\mathrm{i}} \mathrm{q}\right), 1 \leq \mathrm{i} \leq\left\lceil\frac{\mathrm{m}}{2}\right\rceil$ this mean that all ideal vertices of $\mathrm{V}$ are adjacent each other, and $\mathrm{V}$ form $\mathrm{K}_{\left\lceil\frac{\mathrm{m}}{2}\right\rceil}$, since $\left(\mathrm{p}^{\mathrm{m}}\right)$ is adjacent with all vertices in $\mathrm{V}$ and not adjacent with $\left(\mathrm{p}^{\mathrm{i}}\right)$, where $1 \leq \mathrm{i} \leq \mathrm{m}-1$, also $\mathrm{V}_{\left\lceil\frac{\mathrm{m}}{2}\right\rceil}$ not adjacent with every vertices $\left(\mathrm{p}^{\mathrm{j}} \mathrm{q}\right)$, where $1 \leq \mathrm{j} \leq\left\lceil\frac{\mathrm{m}}{2}\right\rceil-1$, therefor $\mathrm{V} \cup\left\{\left(\mathrm{p}^{\mathrm{m}}\right)\right\}$ form the largest sub-graph $\mathrm{K}_{\left\lceil\frac{\mathrm{m}}{2}\right\rceil+1}$ in $\mathrm{AG}\left(\mathrm{Z}_{\mathrm{p}}{ }^{\mathrm{m}} \mathrm{q}\right)$. Then $\omega\left(\mathrm{AG}\left(\mathrm{Z}_{\mathrm{p}}{ }^{\mathrm{m}} \mathrm{q}\right)\right)=\left\lceil\frac{\mathrm{m}}{2}\right\rceil+1$.

\section{Proposition 3.3}

For all positive number $\mathrm{m} \geq 3$, we have $\operatorname{diam}\left(\mathrm{AG}\left(\mathrm{Z}_{\mathrm{P}}{ }^{\mathrm{m}} \mathrm{q}\right)\right)=3$

Proof: In $A G\left(Z_{P} m q\right)$ we can find four ideal vertices are defined as $\alpha_{1}=(p), \alpha_{2}=\left(p^{m-1} q\right)$, $\alpha_{3}=\left(p^{m}\right)$ and $\alpha_{4}=(q)$ since $\operatorname{deg}\left(\alpha_{1}\right)=\operatorname{deg}\left(\alpha_{4}\right), \alpha_{1} \alpha_{2}=0, \alpha_{2} \alpha_{3}=0$ and $\alpha_{3} \alpha_{4}=0$ but $\alpha_{1} \alpha_{3} \neq 0$, $\alpha_{2} \alpha_{4} \neq 0$ and $\alpha_{1} \alpha_{4} \neq 0$ so that $\mathrm{d}\left(\alpha_{1}, \alpha_{4}\right)=3$, Therefor $\operatorname{dim} \operatorname{AG}\left(Z_{\mathrm{P}} \mathrm{q}\right)=3$.

Example: Let $\mathrm{R}=\mathrm{Z}_{24}$, then the diameter of the graph $\mathrm{AG}(\mathrm{R})$ is equal 3. 


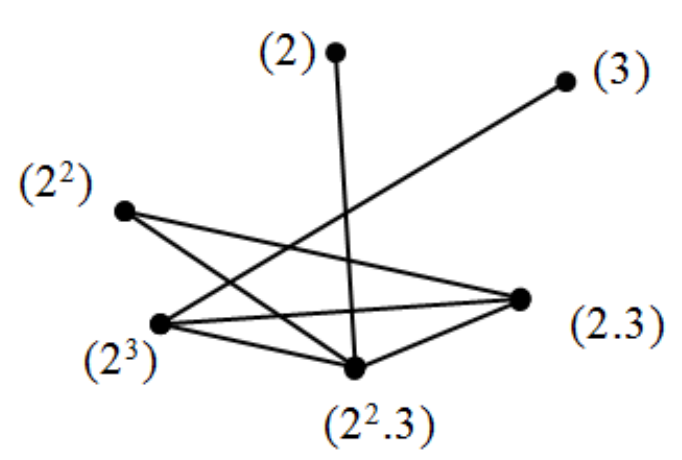

\section{On Annihilating - Ideal Graph of $Z_{p}{ }^{m} q^{r}$}

Let $m$ and $r$ are positive numbers such that $m, r \geq 2$. In this section, we can extended all results in section 3

\section{Theorem4.1}

Let $R=Z_{p}{ }^{m}{ }_{q}^{r}$, then $A G(R)$ has order $m r+m+r-1$ vertices and the sizes.

$a_{1}\left\{\begin{array}{cl}\frac{m^{2} r^{2}+3 m^{2} r+3 m r^{2}-4 m-4 r+2 r^{2}+2 m^{2}}{8}, & \text { if } m \text { and } r \text { are even, } \\ \frac{m^{2} r^{2}+3 m^{2} r+3 m r^{2}-3 m-3 r+2 r^{2}+2 m^{2}+3}{8}, & \text { if } m \text { and } r \text { are odd, } \\ \frac{m^{2} r^{2}+3 m^{2} r+3 m r^{2}-4 m-3 r+2 r^{2}+2 m^{2}+2}{8}, & \text { if m odd and } r \text { even. }\end{array}\right.$

Proof : Since $R$ has ideals $\left(p^{i}\right),\left(q^{k}\right)$ where $1 \leq i \leq m, 1 \leq k \leq r$ and $\left(p^{i} q^{k}\right)$, where $1 \leq i \leq m$ , $1 \leq \mathrm{k} \leq \mathrm{r}$, since $\mathrm{p}^{\mathrm{m}} \mathrm{q}^{\mathrm{r}} \equiv 0\left(\bmod \mathrm{p}^{\mathrm{m}} \mathrm{q}^{\mathrm{r}}\right)$, hence the order of $\mathrm{AG}(\mathrm{R})=\mathrm{mr}+\mathrm{m}+\mathrm{r}-1$.

Now to find the size we must find degree of all vertices ideal of $R$ since $\left(p^{i}\right)$ adjacent with $\left(\mathrm{p}^{\mathrm{j}} \mathrm{q}^{\mathrm{r}}\right)$ iff $\mathrm{i}+\mathrm{j} \geq \mathrm{m}$, then $\operatorname{deg}\left(\mathrm{p}^{\mathrm{i}}\right)=\sum_{\mathrm{j}=\mathrm{m}-\mathrm{i}}^{\mathrm{m}-1} 1=\mathrm{i}$ similarly $\operatorname{deg}\left(\mathrm{q}^{\mathrm{k}}\right)=\mathrm{k}$.

To find deg $\left(p^{i} q^{k}\right)$, where $1 \leq i \leq m, 1 \leq k \leq r$ and $\left(p^{i} q^{k}\right) \neq p^{m} q^{r}$.

Since $\left(p^{i} q^{k}\right)$ adjacent with $\left(p^{j} q^{s}\right)$ iff $i+j \geq m$ and $k+s \geq r$, so that $j=m-i, \ldots, m$ and $s=r-k$ $, \ldots, r$, which implies that $\sum_{\mathrm{j}=\mathrm{m}-\mathrm{i}}^{\mathrm{m}} \sum_{\mathrm{s}=\mathrm{r}-\mathrm{k}}^{\mathrm{r}} 1=(\mathrm{m}-(\mathrm{m}-\mathrm{i})+1)(\mathrm{r}-(\mathrm{r}-\mathrm{k})+1)=$ $(\mathrm{i}+1)(\mathrm{k}+1)$, but $\mathrm{p}^{\mathrm{m}} \mathrm{q}^{\mathrm{r}} \equiv 0\left(\bmod \mathrm{p}^{\mathrm{m}} \mathrm{q}^{\mathrm{r}}\right)$, also $\left(\mathrm{p}^{\mathrm{i}} \mathrm{q}^{\mathrm{k}}\right)$ has loop iff $\mathrm{i} \geq\left\lceil\frac{\mathrm{m}}{2}\right\rceil$ and $\mathrm{k} \geq\left\lceil\frac{\mathrm{r}}{2}\right\rceil$. So that $\operatorname{deg}\left(\mathrm{p}^{\mathrm{i}} \mathrm{q}^{\mathrm{k}}\right)= \begin{cases}(\mathrm{i}+1)(\mathrm{k}+1)-2, & \text { if }\left\lceil\frac{\mathrm{m}}{2}\right\rceil \leq \mathrm{i} \text { and }\left\lceil\frac{\mathrm{r}}{2}\right\rceil \leq \mathrm{k}, \\ (\mathrm{i}+1)(\mathrm{k}+1)-1, & \text { otherwise. }\end{cases}$

Now, we find $\mathrm{a}_{1}$ of $\mathrm{R}$ Since $2 \mathrm{a}_{1}=\sum_{\mathrm{v} \in \mathrm{AG}(\mathrm{R})} \operatorname{deg} \mathrm{v}$, then we have three cases

Case 1: If $m$ and $r$ are even

$$
\begin{gathered}
2 \mathrm{a}_{1}=\sum_{\mathrm{v} \in A \mathrm{G}(\mathrm{R})} \operatorname{deg} \mathrm{V}=\sum_{\mathrm{i}=1}^{\mathrm{m}} \sum_{\mathrm{k}=1}^{\mathrm{r}}((\mathrm{i}+1)(\mathrm{k}+1)-1)-\sum_{\mathrm{i}=\frac{\mathrm{m}}{2} \mathrm{k}=\frac{\mathrm{r}}{2}}^{\mathrm{r}} 1-((\mathrm{m}+1)(\mathrm{r}+1)-2)+\sum_{\mathrm{i}=1}^{\mathrm{m}} \mathrm{i}+\sum_{\mathrm{k}=1}^{\mathrm{r}} \mathrm{k} \\
=\sum_{\mathrm{i}=1}^{\mathrm{m}}(\mathrm{i}+1) \sum_{\mathrm{k}=1}^{\mathrm{r}}(\mathrm{k}+1)-\sum_{\mathrm{i}=1}^{\mathrm{m}} \sum_{\mathrm{k}=1}^{\mathrm{r}} 1-\sum_{\mathrm{i}=\frac{\mathrm{m}}{2}}^{\mathrm{m}} \sum_{\mathrm{k}=\frac{\mathrm{r}}{2}}^{\mathrm{r}} 1-((\mathrm{m}+1)(\mathrm{r}+1)-2)+\sum_{\mathrm{i}=1}^{\mathrm{m}} \mathrm{i}+\sum_{\mathrm{k}=1}^{\mathrm{r}} \mathrm{k} \\
=\left(\frac{\mathrm{m}(\mathrm{m}+1)}{2}+\mathrm{m}\right)\left(\frac{\mathrm{r}(\mathrm{r}+1)}{2}+\mathrm{r}\right)-\mathrm{mr}-\frac{\mathrm{mr}+2 \mathrm{~m}+2 \mathrm{r}+4}{4}-(\mathrm{mr}+\mathrm{m}+\mathrm{r}-1)+\frac{\mathrm{m}(\mathrm{m}+1)}{2}+\frac{\mathrm{r}(\mathrm{r}+1)}{2} \\
=\frac{\mathrm{m}^{2} \mathrm{r}^{2}+3 \mathrm{~m}^{2} \mathrm{r}+3 \mathrm{~m} \mathrm{r}^{2}-6 \mathrm{~m}-6 \mathrm{r}+2 \mathrm{r}^{2}+2 \mathrm{r}+2 \mathrm{~m}^{2}+2 \mathrm{~m}}{4}
\end{gathered}
$$


So that

$$
\mathrm{a}_{1}=\frac{\mathrm{m}^{2} \mathrm{r}^{2}+3 \mathrm{~m}^{2} \mathrm{r}+3 \mathrm{~m} \mathrm{r}^{2}-4 \mathrm{~m}-4 \mathrm{r}+2 \mathrm{r}^{2}+2 \mathrm{~m}^{2}}{8}
$$

Case 2:If $m$ and $r$ are odd we get

$$
\begin{gathered}
2 \mathrm{a}_{1}=\sum_{\mathrm{v} \in \mathrm{AG}(\mathrm{k})} \mathrm{deg} \mathrm{V}=\sum_{\mathrm{i}=1}^{\mathrm{m}}(\mathrm{i}+1) \sum_{\mathrm{k}=1}^{\mathrm{r}}(\mathrm{k}+1)-\sum_{\mathrm{i}=1}^{\mathrm{m}} \sum_{\mathrm{k}=1}^{\mathrm{r}} 1-\sum_{\mathrm{i}=\frac{\mathrm{m}+1}{2}}^{\mathrm{m}} \sum_{\mathrm{k}=\frac{\mathrm{r}+1}{2}}^{\mathrm{r}} 1-((\mathrm{m}+1)(\mathrm{r}+1)-2)+\sum_{\mathrm{i}=1}^{\mathrm{m}} \mathrm{i}+\sum_{\mathrm{k}=1}^{\mathrm{r}} \mathrm{k} \\
=\left(\sum_{i=1}^{\mathrm{m}} i+\sum_{i=1}^{m} 1\right)\left(\sum_{k=1}^{r} k+\sum_{k=1}^{r} 1\right)-r m-\left(\frac{r+1}{2}\right)\left(\frac{m+1}{2}\right)-((m+1)(r+1)-2)+\sum_{i=1}^{m} i+\sum_{k=1}^{r} k \\
=\frac{\mathrm{m}^{2} \mathrm{r}^{2}+3 \mathrm{~m}^{2} \mathrm{r}+3 \mathrm{~m} \mathrm{r}^{2}-5 \mathrm{~m}-5 \mathrm{r}+3+2 \mathrm{r}^{2}+2 \mathrm{r}+2 \mathrm{~m}^{2}+2 \mathrm{~m}}{4}
\end{gathered}
$$

So that

$$
a_{1}=\frac{m^{2} r^{2}+3 m^{2} r+3 m r^{2}-3 m-3 r+2 r^{2}+2 m^{2}+3}{8}
$$

Case 3: Since $p$ and $q$ are distinct prime number, then if $m$ odd and $r$ even or $r$ odd and $\mathrm{m}$ even we get the same result, so without loss generality let $\mathrm{m}$ odd and $\mathrm{r}$ even.

$$
\begin{gathered}
2 \mathrm{a}_{1}=\sum_{\mathrm{v} \in A \mathrm{G}^{(}(\mathrm{R})} \operatorname{deg} \mathrm{V}=\sum_{\mathrm{i}=1}^{\mathrm{m}}(\mathrm{i}+1) \sum_{\mathrm{k}=1}^{\mathrm{r}}(\mathrm{k}+1)-\sum_{\mathrm{i}=1}^{\mathrm{m}} \sum_{\mathrm{k}=1}^{\mathrm{r}} 1-\sum_{\mathrm{i}=\frac{\mathrm{m}+1}{2}}^{\mathrm{m}} \sum_{\mathrm{k}=\frac{\mathrm{r}}{2}}^{\mathrm{r}} 1-((\mathrm{m}+1)(\mathrm{r}+1)-2)+\sum_{\mathrm{i}=1}^{\mathrm{m}} \mathrm{i}+\sum_{\mathrm{k}=1}^{\mathrm{r}} \mathrm{k} \\
\left(\sum_{\mathrm{i}=1}^{\mathrm{m}} \mathrm{i}+\sum_{\mathrm{i}=1}^{\mathrm{m}} 1\right)\left(\sum_{\mathrm{k}=1}^{\mathrm{r}} \mathrm{k}+\sum_{\mathrm{k}=1}^{\mathrm{r}} 1\right)-\mathrm{rm}-\left(\frac{\mathrm{m}+1}{2}\right)\left(\frac{\mathrm{r}+2}{2}\right)-(\mathrm{m}+1)(\mathrm{r}+1)-2+\sum_{\mathrm{i}=1}^{\mathrm{m}} \mathrm{i}+\sum_{\mathrm{k}=1}^{\mathrm{r}} \mathrm{k} \\
=\frac{\mathrm{m}^{2} \mathrm{r}^{2}+3 \mathrm{~m}^{2} \mathrm{r}+3 \mathrm{~m} \mathrm{r}^{2}-6 \mathrm{~m}-5 \mathrm{r}+2+2 \mathrm{r}^{2}+2 \mathrm{r}+2 \mathrm{~m}^{2}+2 \mathrm{~m}}{4}
\end{gathered}
$$

So that

$a_{1}=\frac{m^{2} r^{2}+3 m^{2} r+3 m r^{2}-4 m-3 r+2 r^{2}+2 m^{2}+2}{8}$.

\section{Proposition 4.2}

For all positive numbers $m, r \geq 2, \operatorname{diam}\left(A G\left(Z_{P}{ }^{m} q^{r}\right)\right)=3$.

Proof: By the same method of proof Proposition 3.3, we can choose $\alpha_{1}=(p)$ and $\alpha_{2}=(q)$ and we get $d\left(\alpha_{1}, \alpha_{2}\right)=3$.

\section{Theorem 4.3}

Let $R=Z_{p}{ }^{m} q^{r}$, then $A G(R)$ has a sub-graph of $K_{s}$, Furthermore $\omega(A G(R)=s$, where

$$
s= \begin{cases}\frac{m r+2 m+2 r}{4}, & \text { if } m \text { and } r \text { are even }, \\ \frac{m r+m+r+1}{4}, & \text { if } m \text { and } r \text { are odd }, \\ \frac{m r+m+2(r+1)}{4}, & \text { if } m \text { even and } r \text { odd. }\end{cases}
$$


Proof: If $m$ and $r$ are even, then $v_{i j}=\left(p^{i} q^{j}\right)$ vertices, where $m / 2 \leq i \leq m, r / 2 \leq j \leq r$ and $\mathrm{v}_{\mathrm{ij}} \neq\left(\mathrm{p}^{\mathrm{m}} \mathrm{q}^{\mathrm{r}}\right)=0$ are all adjacent with every others. And the number of this vertices equal $\sum_{m / 2}^{m} \sum_{r / 2}^{r} 1=\frac{m r+2 m+2 r}{4}=s$.

.Also any vertices in $A G(R) /\left\{v_{i j}\right\}$ are non-adjacent with $\left(\mathrm{p}^{\mathrm{m} / 2} \mathrm{q}^{\mathrm{r} / 2}\right)$, therefore $\mathrm{K}_{\mathrm{s}}$ the largest sub-graph in $A G(R)$ in this case. Then $\omega(A G(R))=s$.

If $\mathrm{m}$ and $\mathrm{r}$ are odd, similarly $\mathrm{v}_{\mathrm{ij}}=\left(\mathrm{p}^{\mathrm{i}} \mathrm{q}^{\mathrm{j}}\right)$, where $(\mathrm{m}+1) / 2 \leq \mathrm{i} \leq \mathrm{m}, \quad(\mathrm{r}+1) / 2 \leq \mathrm{j} \leq \mathrm{r}$ and $\mathrm{v}_{\mathrm{ij}} \neq\left(\mathrm{p}^{\mathrm{m}} \mathrm{q}^{\mathrm{r}}\right)=0$ with the vertices $\left(\mathrm{p}^{(\mathrm{m}+1) / 2} \mathrm{q}^{(\mathrm{r}-1) / 2}\right)$ and $\left(\mathrm{p}^{(\mathrm{m}-1) / 2} \mathrm{q}^{(\mathrm{r}+1) / 2}\right)$ the largest sub-graph in $A G(R)$. And the number of vertices equal

$\mathrm{s}=\frac{\mathrm{mr}+\mathrm{m}+\mathrm{r}+1}{4}$

Similarly, if m even and $r$ odd, we get

$\mathrm{s}=\frac{\mathrm{mr}+\mathrm{m}+2(\mathrm{r}+1)}{4}$.

\section{Hosoya polynomial and Wiener index of Annihilating -Ideal graph of $\mathbf{Z}_{\mathbf{n}}$.}

"Hosoya polynomial of the graph $G$ is defined by : $H(G ; x)=\sum_{k=0}^{\operatorname{diam}(G)} d(G, k) x^{k}$, where $d(G, k)$ the number of pairs of vertices of a graph $G$ are at distance $k$ a part, for $\mathrm{k}=0,1, \ldots, \operatorname{diam}(\mathrm{G})$. The Winer index of $\mathrm{G}$ is define as the sum of all distances between vertices of the graph and denoted by $\mathrm{W}(\mathrm{G})$, and we can find this index by differentiating Hosoya polynomial with respect to $x$ then putting $x=1 "$, see[5], [8].

In [1] Ahmadi and Jahani-Nezhad first study the Winer index of zero divisor graph of $Z_{n}$ where $n=p^{2}$ and $p^{2} q$. In [6] Mohammad and Authman extended this result for $\mathrm{n}=\mathrm{p}^{\mathrm{m}}$ and $\mathrm{p}^{\mathrm{m}} \mathrm{q}$ and study the Hosoya polynomial of this type. In this section we study Hosoya polynomial and Winer index of annihilating-ideal graph of $Z_{n}$, where $n=p^{m}, p^{m} q$ and $\mathrm{p}^{\mathrm{m}} \mathrm{q}^{\mathrm{r}}$.

\section{Lemma 5.1[5]}

Let $\mathrm{G}$ be a connected graph of order $r$. Then $\sum_{\mathrm{i}=0}^{\operatorname{diam}(G)} \mathrm{d}(\mathrm{G}, \mathrm{i})=\frac{1}{2} \mathrm{r}(\mathrm{r}+1)$.

Clearly if $R=Z_{p}^{2}$, then $A G(R)=K_{1}$, therefore $H\left(A G(R, x)=1\right.$. Also if $R=Z_{p}{ }^{s}$, where $s=3,4$, then $A G(R)=K_{2}$ or $K_{1,2}$ respectively so that $H\left(A G(R, x)=2+x\right.$ or $3+2 x+x^{2}$ respectively. Therefore we calculate Hosoya polynomial of $A G\left(Z_{p}{ }^{m}\right)$ for all positive number $\mathrm{m} \geq 4$.

\section{Theorem 5.2}

For all positive number $\mathrm{m} \geq 4, \mathrm{H}\left(\mathrm{AG}\left(\mathrm{Z}_{\mathrm{p}}{ }^{\mathrm{m}}, \mathrm{x}\right)=\mathrm{a}_{0}+\mathrm{a}_{1} \mathrm{x}+\mathrm{a}_{2} \mathrm{x}^{2}\right.$, where $\mathrm{a}_{0}=\mathrm{m}-1$,

$$
\begin{aligned}
& \mathrm{a}_{1}=\left\{\begin{array}{l}
\frac{\mathrm{m}(\mathrm{m}-2)}{4}, \text { if m even, } \\
\frac{(\mathrm{m}-1)^{2}}{4}, \text { if m odd, }
\end{array}\right. \text { and } \\
& \mathrm{a}_{2}=\left\{\begin{array}{l}
\frac{(\mathrm{m}-2)^{2}}{4}, \text { if m even, } \\
\frac{\mathrm{m}^{2}-4 \mathrm{~m}+3}{4}, \text { if m odd. }
\end{array}\right.
\end{aligned}
$$

Proof: From Theorem2.1 and Lemma5.1, we get the result. 


\section{Corollary 5.3}

For all positive number $\mathrm{m} \geq 4, \mathrm{~W}\left(\mathrm{AG}\left(\mathrm{Z}_{\mathrm{p}}{ }^{\mathrm{m}}\right)= \begin{cases}\frac{(\mathrm{m}-2)(3 \mathrm{~m}-4)}{4}, & \text { if } \mathrm{m} \text { even, } \\ \frac{(\mathrm{m}-1)(3 \mathrm{~m}-7)}{4}, & \text { if } \mathrm{m} \text { odd. }\end{cases}\right.$

Example 1:

Let $\mathbf{R}=\mathrm{Z}_{\mathrm{p}}{ }^{6}$ then

$\mathrm{H}\left(\mathrm{AG}\left(\mathrm{Z}_{\mathrm{p}}{ }^{6}, \mathrm{x}\right)=5+6 \mathrm{x}+4 \mathrm{x}^{2}\right.$
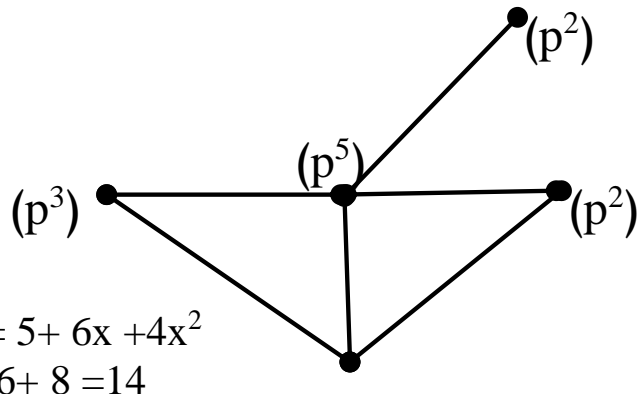

$\mathbf{W}\left(\mathrm{AG}\left(\mathrm{Zp}^{6}\right)\right)=6+8=14$

\section{Theorem 5.4}

For all positive number $m \geq 3$, we have

$$
H\left(A G\left(Z_{p^{m} q}, x\right)=\left\{\begin{array}{cl}
2 m+\frac{3 m^{2}}{4} x+\frac{5 m^{2}-8 m+4}{4} x^{2}+(m-1) x^{3}, & \text { if } m \text { even, } \\
2 m+\frac{3 m^{2}+1}{4} x+\frac{5 m^{2}-8 m+3}{4} x^{2}+(m-1) x^{3}, & \text { if } m \text { odd. }
\end{array}\right.\right.
$$

Proof: Since $\operatorname{diam}\left(A G\left(Z_{p}{ }_{q}\right)=3\right.$ and applying Theorem3.1, we have $a_{0}=2 \mathrm{~m}$ and .

$a_{1}= \begin{cases}\frac{3 m^{2}}{4}, & \text { if m even, } \\ \frac{3 m^{2}+1}{4}, & \text { if m odd. }\end{cases}$

Now to find $a_{3}$ we can write $A G\left(Z_{p}{ }_{q}\right)=\bigcup_{i=1}^{m}\left(B_{i} \cup C_{i}\right)$, where $B_{i}=\left(p^{m-i} q\right)$, $\mathrm{i}=1,2, \ldots, \mathrm{m}$ and $\mathrm{C}_{\mathrm{i}}=\left(\mathrm{p}^{\mathrm{m}-\mathrm{i}+1}\right), \mathrm{i}=1,2, \ldots, \mathrm{m}$. Then there are three cases.

Case 1: Let $x \in B_{i}$ and $y \in B_{j}$ where $1 \leq \mathrm{i}, j \leq m$ then $C_{1}=\left(P^{m}\right)$ is adjacent with every vertices in $B_{i} 1 \leq i \leq m$, and that means $d(x, y) \leq 2$ and this contradict our hypothesis.

Case 2: Let $x \in C_{i}$ and $y \in C j$ where $1 \leq \mathrm{i}, j \leq m$ we see that a vertex $B_{i}=\left(p^{m-1} q\right)$ is adjacent with every vertices in $C_{i}$ for all $1 \leq \mathrm{i} \leq \mathrm{m}$ because $1+\mathrm{i} \leq 1+\mathrm{m}$ for any $1 \leq \mathrm{i} \leq \mathrm{m}$ , and that means $\mathrm{d}(\mathrm{x}, \mathrm{y}) \leq 2$ and this contradiction.

Case 3: If $x \in B_{i}$ and $y \in C_{j}$ for some $1 \leq i, j \leq m$, in this case we see that $d(x, y)=3$ if and only if $\mathrm{i}=\mathrm{m}$ and $2 \leq \mathrm{j} \leq \mathrm{m}$, because that $\mathrm{d}(\mathrm{x}, \mathrm{y}) \leq 2$ for any $1 \leq \mathrm{i} \leq \mathrm{m}-1$ and 2 $\leq \mathrm{j} \leq \mathrm{m}$ also $\mathrm{d}(\mathrm{x}, \mathrm{y})=1$ for $1 \leq \mathrm{i} \leq \mathrm{m}$ and $\mathrm{j}=1$, therefor the number of of pairs of vertices that that are distance three apart is (m-1).

Finally, we find $\mathrm{a}_{2}$, applying by Lemma 5.1 we get $\mathrm{a}_{2}=\left(5 \mathrm{~m}^{2}-8 \mathrm{~m}+4\right) / 4$, when $\mathrm{m}$ even, $\mathrm{a}_{2}=\left(5 \mathrm{~m}^{2}-8 \mathrm{~m}+3\right) / 4$, when $\mathrm{m}$ odd

\section{Corollary 5.5:}

$$
W\left(\operatorname{AG}\left(Z_{\mathrm{p}^{\mathrm{m}} \mathrm{q}}\right)\right)=\left\{\begin{array}{l}
\frac{13 \mathrm{~m}^{2}-4 \mathrm{~m}-4}{4}, \text { if } m \text { even, } \\
\frac{13 \mathrm{~m}^{2}-4 \mathrm{~m}-5}{4}, \text { if } \mathrm{m} \text { odd. }
\end{array}\right.
$$

\section{Example 2:}

Let $\quad \mathrm{R}=\mathrm{Z}_{\mathrm{P}}{ }_{\mathrm{q}}{ }_{\mathrm{q}}$, then $\mathrm{H}\left(\mathrm{AG}\left(\mathrm{Z}_{\mathrm{P}}{ }_{\mathrm{q}}, \mathrm{x}\right)\right)=12+27 \mathrm{x}+34 \mathrm{x}^{2}+5 \mathrm{x}^{3}$, $\mathrm{W}\left(\mathrm{AG}\left(\mathrm{Z}_{\mathrm{P}}{ }_{\mathrm{q}}\right)\right)=27+2.34+3.5=110$ 


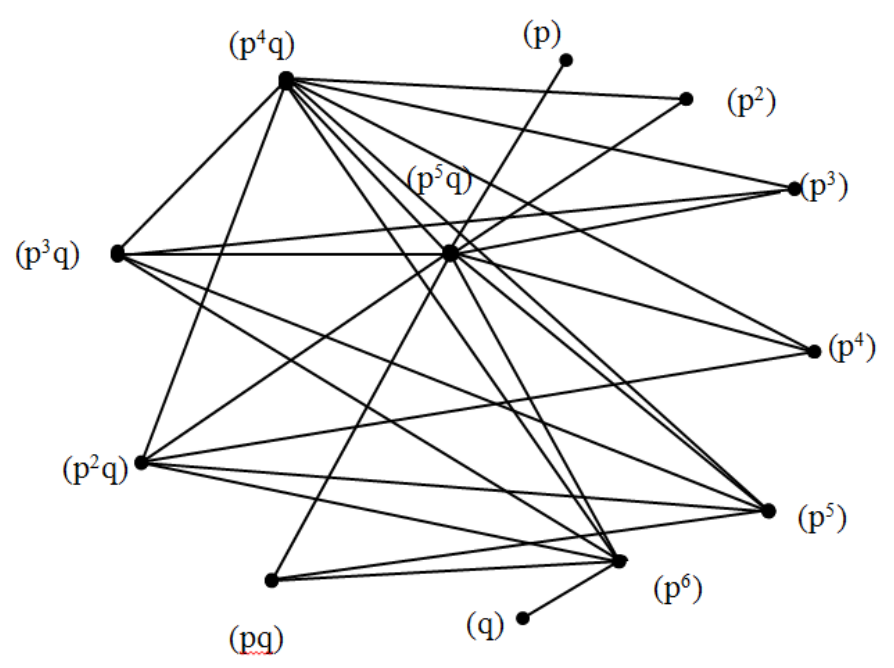

Finally we give extended to theorem 5.4

\section{Theorem 5.6}

For any positive numbers $m, r \geq 2$, we have

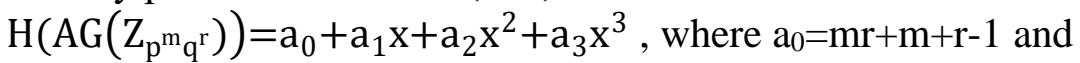

$$
\begin{aligned}
& a_{1}=\left\{\begin{array}{l}
\frac{m^{2} r^{2}+3 m^{2} r+3 m r^{2}-4 m-4 r+2 r^{2}+2 m^{2}}{8}, \text { if } m \text { and } r \text { are even, } \\
\frac{m^{2} r^{2}+3 m^{2} r+3 m r^{2}-3 m-3 r+2 r^{2}+2 m^{2}+3}{8}, \text { if } m \text { and } r \text { are odd, } \\
\frac{m^{2} r^{2}+3 m^{2} r+3 m r^{2}-4 m-3 r+2 r^{2}+2 m^{2}+2}{8}, \text { if } m \text { odd and } r \text { even, }
\end{array}\right. \\
& a_{2}=\left\{\begin{array}{l}
\frac{3 m^{2} r^{2}+5 m^{2} r+5 m r^{2}+2 m^{2}+2 r^{2}-12 m r-8 m-8 r+16}{8}, \text { if } m \text { and } r \text { even, } \\
\frac{3 m^{2} r^{2}+5 m^{2} r+5 m r^{2}+2 m^{2}+2 r^{2}-12 m r-9 m-9 r+13}{8}, \text { if } m \text { and } r \text { odd, } \\
\frac{3 m^{2} r^{2}+5 m^{2} r+5 m r^{2}+2 m^{2}+2 r^{2}-12 m r-8 m-9 r+14}{8}, \text { if m odd and } r \text { even. }
\end{array}\right.
\end{aligned}
$$

and $\mathrm{a}_{3}=\mathrm{mr}-1$

Proof:_By Theorem 4.1, AG(R) has order $a_{0}=m r+m+r-1$ vertices and the sizes.

$$
a_{1}=\left\{\begin{array}{l}
\frac{m^{2} r^{2}+3 m^{2} r+3 m r^{2}-4 m-4 r+2 r^{2}+2 m^{2}}{8}, \text { if } m \text { and } r \text { are even, } \\
\frac{m^{2} r^{2}+3 m^{2} r+3 m r^{2}-3 m-3 r+2 r^{2}+2 m^{2}+3}{8}, \text { if } m \text { and } r \text { are odd, } \\
\frac{m^{2} r^{2}+3 m^{2} r+3 m r^{2}-4 m-3 r+2 r^{2}+2 m^{2}+2}{8}, \text { if m odd and } r \text { even, }
\end{array}\right.
$$

To find a3 let $A=\left\{\left(p^{i} q^{j}\right): 1 \leq i \leq m\right.$ and, $\left.1 \leq j \leq r\right\}-\{0\}, B=\left\{\left(p^{i}\right): 1 \leq \mathrm{i} \leq \mathrm{m}\right\}$ and $\mathrm{C}=\left\{\left(\mathrm{q}^{\mathrm{j}}\right): 1 \leq \mathrm{j} \leq \mathrm{r}\right\}$.

If $x, y \in A$ or $B$, since every element in this case is adjacent with ideal vertex $\left(p^{m-1} q^{r}\right)$ so that $\mathrm{d}(\mathrm{x}, \mathrm{y}) \leq 2$ which is contradiction .

Similarly, if $\mathrm{x}, \mathrm{y} \in \mathrm{A}$ or $\mathrm{C}$, then every element in this case is adjacent with ideal vertex $\left(\mathrm{p}^{\mathrm{m}} \mathrm{q}^{\mathrm{r}-1}\right)$ so that $\mathrm{d}(\mathrm{x}, \mathrm{y}) \leq 2$ which is contradiction, if $\mathrm{x} \in \mathrm{B}$ and $\mathrm{y} \in \mathrm{C}$ since every element 
in $B$ is adjacent with ideal vertex $\left(p^{i} q^{r}\right)$ for $i=1, \ldots, m-1$ and therefor $d(x, y)=3$ except the case where $i=m$ and $j=r$ so that the number of pairs of vertex that are distance three a part is $\mathrm{mr}-1$.

To find $\mathrm{a}_{2}$, since $\sum_{\mathrm{i}=0}^{\operatorname{diam}(\mathrm{G})} \mathrm{d}(\mathrm{G}, \mathrm{i})=\frac{\mathrm{r}(\mathrm{r}+1)}{2}$ by Lemma 5.1, then

$$
a_{2}=\left\{\begin{array}{c}
\frac{3 m^{2} r^{2}+5 m^{2} r+5 m r^{2}+2 m^{2}+2 r^{2}-12 m r-8 m-8 r+16}{8}, \text { if } m \text { and } r \text { even, } \\
\frac{3 m^{2} r^{2}+5 m^{2} r+5 m r^{2}+2 m^{2}+2 r^{2}-12 m r-9 m-9 r+13}{8}, \text { if } m \text { and } r \text { odd, } \\
\frac{3 m^{2} r^{2}+5 m^{2} r+5 m r^{2}+2 m^{2}+2 r^{2}-12 m r-8 m-9 r+14}{8}, \text { if m odd and } r \text { even. }
\end{array}\right.
$$

\section{Corollary 5.7:}

$W\left(A G\left(Z_{p^{m}} q^{r}\right)\right)=\left\{\begin{array}{cl}\frac{7 m^{2} r^{2}-20 m-20 r+13 m^{2} r+13 m r^{2}+6 r^{2}+6 m^{2}+8}{8} & \text { if } m \text { and } r \text { even } \\ \frac{7 m^{2} r^{2}-21 m-21 r+13 m^{2} r+13 m r^{2}+6 r^{2}+6 m^{2}+5}{8} & \text { if } m \text { and } r \text { odd } \\ \frac{7 m^{2} r^{2}-20 m-21 r+13 m^{2} r+13 m r^{2}+6 r^{2}+6 m^{2}+6}{8} & \text { if } m \text { odd and } r \text { even }\end{array}\right.$ 


\section{REFERENCES}

[1] M. R. Ahmadi and R. Jahani-Nezhad, "Energy and Wiener Index of Zero-Divisor Graphs," Iranian Journal of Mathematical Chemistry, vol. 2, pp. 45-51, 2011.

[2] M. Behboodi and Z. Rakeei, "The annihilating-ideal graph of commutative rings I", Journal of Algebra and its Applications, vol. 10, pp. 727-739, 2011.

[3] G. Chartrand and L. Lesniak, Graphs and Digraphs, Wadsworth Inc. 1996.

[4] K. H. Rosen, Elementary number theory and its applications: AddisonWesley, 1993.

[5] H. Hosoya, "On some counting polynomials in chemistry", Discrete Applied Mathematics, vol. 19, pp. 239-257, 1988.

[6] H. Q. Mohammad and M. N. Authman, "Hosoya polynomial and Wiener index of zero-divisor graph of $\mathrm{Z}_{\mathrm{n}}$ ",accepted in Al-Rafidain Journal of computer scinces and mathematics.", 2014.

[7] H. Q. Mohammad and S. A. Younus, "Planar Annihilating-Ideal Graph of Commutative Rings", International Journal of Enhanced Research in Science, Technology \& Engineering, vol. 6, pp. 85-92, 2017.

[8] H. Wiener, "Structural determination of paraffin boiling points," Journal of the American Chemical Society, vol. 69, pp. 17-20, 1947. 